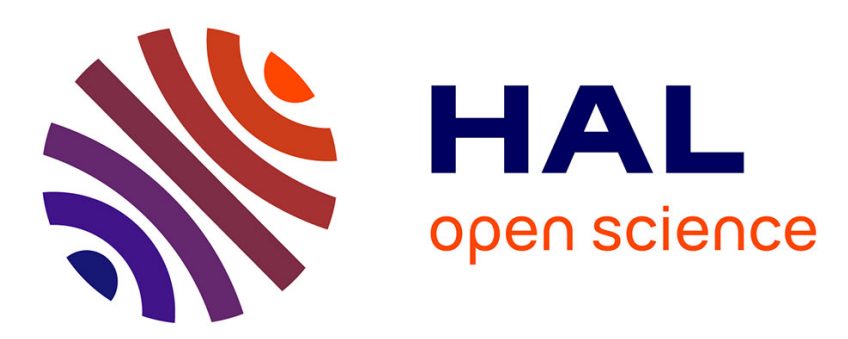

\title{
Le rituel de Walkui (KBo 32.176): quelques réflexions sur ladéesse de la nuit et l'image du porc dans le monde hittite.
}

Alice Mouton

\section{To cite this version:}

Alice Mouton. Le rituel de Walkui (KBo 32.176): quelques réflexions sur ladéesse de la nuit et l'image du porc dans le monde hittite.. Zeitschr. f. Assyriologie Bd, 2004, 94, pp.85-105. halshs-00363601

\section{HAL Id: halshs-00363601 https://shs.hal.science/halshs-00363601}

Submitted on 23 Feb 2009

HAL is a multi-disciplinary open access archive for the deposit and dissemination of scientific research documents, whether they are published or not. The documents may come from teaching and research institutions in France or abroad, or from public or private research centers.
L'archive ouverte pluridisciplinaire HAL, est destinée au dépôt et à la diffusion de documents scientifiques de niveau recherche, publiés ou non, émanant des établissements d'enseignement et de recherche français ou étrangers, des laboratoires publics ou privés. 


\title{
Le rituel de Walkui (KBo 32.176): quelques réflexions sur la déesse de la nuit et l'image du porc dans le monde hittite ${ }^{1}$
}

\author{
par Alice Mouton - Paris/Leyde
}

1. Walkui is a priest of the deity called DINGIR $\mathrm{GE}_{6}$. This latter has to be identified with the "goddess of the night», a kind of Šaušga. She is probably a star - or a deity represented by a star -, like her Mesopotamian homonyms the «gods of the night».

2. Walkui's ritual seems to refer to the impurity of pork meat. The ancient Anatolian image of the pig has two antinomic facets: on the one hand, this animal is related to the notion of fertility and is bred and eaten by a part of the Hittite population. On the other hand, as a creature linked to the Netherworld, the pig is regarded as impure and seems taboo in some circumstances - at least in Kizzuwatna where Walkui's ritual comes from.

Le texte hittite KBo 32.176 (Bo 83/902) a été découvert dans le temple 12 de la ville haute de Hattuša. Il décrit le rituel de Walkui, un prêtre de la «divinité de la nuit» (DINGIR ${ }^{\mathrm{LIM}}$ MUSI) $)^{2}$ et a été édité par R. Lebrun (1999). Ce dernier a très justement indiqué la datation de ce texte, à savoir moyen-hittite.

${ }^{1}$ Cet article est une version plus développée de ma contribution au Vème Congrès International d'Hittitologie (Çorum, 2-6 Septembre 2002) intitulée «Walkui's Ritual». J'ai eu l'occasion d'étudier le rituel de Walkui dans le cadre de mon doctorat intitulé «Le rêve au Proche-Orient au IIème millénaire av. J.-C.: étude des sources hittites mises en perspective avec le reste du Proche-Orient ancien». Cette thèse de doctorat est préparée sous la direction du Pr. K. R. Veenhof (Université de Leyde) et du Pr. J.-M. Durand (Ecole Pratique des Hautes Etudes, Paris), ainsi que sous la supervision du Pr. J. de Roos que je remercie ici pour ses précieux conseils concernant le rituel de Walkui.

Je suis également reconnaissante envers les Dr. O. Soysal, B. Lion et C. Michel qui ont bien voulu relire cet article. Je tiens à signaler que toute erreur présente dans ce dernier, qu'elle soit philologique ou d'une quelqu'autre nature, ne doit être imputée qu'à moi-même.

Afin de compléter cet article, j'ai recouru aux données du CHD (lui-même soutenu par le «National Endowment for the Humanities») lors de mon séjour à l'Oriental Institute en tant qu'associée au projet. J'ai, pour ce faire, bénéficié d'une bourse Lavoisier du Ministère des Affaires Etrangères français.

2 Il est à noter que le nom de Walkui est aussi mentionné dans la tablette-catalogue KUB 8.71 Vo 7-12 (et duplicat KUB 55.24: 2'-12') qui décrit un rituel devant être pratiqué lorsque quelqu'un voit la divinité de la nuit en rêve. Cette dernière est donc à deux reprises associée au phénomène du rêve. 
KBo 32.176 débute de la manière suivante (Ro 1-6):

1. UMMA I walkui LÚSANGA Š DINGIR ${ }^{L M}$ MUŚ mān anduwahh[aś]

2. zašhiya ururan ${ }^{\mathrm{SAR}}$ našma UZU.ŠAH ezzazi našma

3. parā handanni=ma ANA UZU.ŠAH anda tarnattari $[\varnothing]$

4. našma kan INA É.DINGIR ${ }^{L M}$ anda parā handanni ANA $\mathrm{SAR}^{\mathrm{H}}\left[{ }^{\mathrm{A}}\right]$

5. ururan $^{\mathrm{SAR}}$ anda uemiyezzi nu zurkiya IŠTU $\mathrm{K}\left[\mathrm{U}_{6}\right]$

6. špan [ti $\left.{ }^{3}\right]$ arnamitti $=m a=z a$ IŠTU BA.BA.ZA

«Ainsi (parle) Walkui, prêtre de la divinité de la nuit: si une personn[e] mange dans (son) rêve une plante urura ou de la viande de porc, (si) dans la providence divine de la viande de porc est laissée, (ou si) dans la providence divine (cette personne) trouve dans le temple une plante urura parmi les autres plantes, (alors) elle fe[ra] une offrande de zurkiya avec du pois[son]. Elle arnami ${ }^{4}$ avec de la purée d'orge ${ }^{5} . »$

Qui est la divinité de la nuit dont Walkui est le prêtre? A quoi ressemble-t-elle et quelles sont ses fonctions? C'est ce que nous tenterons de préciser en premier lieu. Puis nous essaierons de comprendre la raison d'être du rituel de Walkui, ce qui revient à examiner la manière dont le porc - et plus particulièrement sa viande - était appréhendé dans le monde hittite.

3 Le colophon emploie la première personne du singulier (šipantahhi). Toutefois, l'incipit semble utiliser la troisième personne du singulier, car la forme verbale arnamitti est à la troisième personne du singulier et semble aller de paire avec sipant-.

4 arnamitti est une $3^{\text {ème }}$ pers. sg. présent du verbe arnami- qui désigne probablement une action rituelle, à l'instar de sipant- (HED I, 162). arnamitti est en effet toujours associé à ce dernier verbe dans les textes. Il apparaît dans les sources suivantes:

1) KUB 29.4+ § 13: 33 f. (Kronasser 1963, 16): EGIR-ŠU־ma MÁš TUR našma SILA $_{4}$ šipandanzi / $n u$ EN SISKUR ${ }_{2}$ arnaminti («Ensuite, on sacrifie un chevreau ou un agneau et

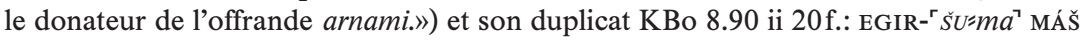
TUR našma SILA $_{4}$ ši pan- ...

] / arnamitti.

2) KUB 29.7 Vo 63 (CTH 480: rituel de purification. Lebrun 1976, 125 et 132, 1. 72): EGIR-anda:ma zurkiyaš UzU zeyantit šipanti namma arnamitti («Ensuite, il [= le donateur de l'offrande EN SISKUR ${ }_{2}$ mentionné ligne 71] fait une offrande de zurkiya avec de la viande cuite puis il arnami.»).

3) Bo 4951 Vo 12 (HED I, 162): ... šipanti nuะza arnamitti

Notre passage, šipanti arnamitti=ma $z a$, équivaut à l'extrait $\mathrm{n}^{\circ} 3$ ). arnami- est un verbe intransitif, contrairement à sipant-. Kronasser $(1963,17)$ propose de traduire arnamitti par «macht sich ... bereit», tout comme J. Tischler qui le traduit par «sich fertig machen» (HEG I, 64). J. Puhvel suggère que ce verbe provienne de la même racine qu'arnu- «make removal, clear away» (HED I, 162). Il reste cependant délicat d'établir le sens exact de ce verbe.

5 Pour la légère variante présente dans le colophon du texte, voir Lebrun (1999). 


\section{L'identité de la déesse de la nuit}

Avant que ce rituel soit mis au jour, la lecture du nom de la divinité de la nuit était l'objet de controverses. En effet, étant donné que son nom était toujours écrit DINGIR $\mathrm{GE}_{6}$, il n'était pas possible de savoir s'il fallait le lire «divinité noire» (Kronasser 1963) ou «divinité de la nuit». O. Carruba avait déjà suggéré que cette dernière interprétation fût la plus appropriée (R1A 3, 355). Le texte KBo 2.8 i 17-18 fournit une première clé de lecture du nom de la divinité en le notant sous la forme DINGIR ${ }^{L M}$ $\mathrm{GE}_{6}{ }^{S I}$, ce qui équivaut à DINGIR ${ }^{L U M} M U S \check{I}$. KBo 32.176 confirme à présent cette interprétation. Le fait que cette divinité soit une déesse fut mis en évidence par H. Kronasser $(1963,40)$.

Le compte-rendu oraculaire KBo 16.97 Vo 12-32 mentionne tour à tour Šaušga de Ninive, la déesse de la nuit de Šamuha, celle de Lahhurama, Šaušga de Hattarina, etc. puis vient la formule «quelque autre Šaušga» (tamaǐ̌̌ma kuiški $\left.{ }^{\mathrm{D} I S ̌ T A R}\right)^{6}$. Par conséquent, la déesse de la nuit est un aspect kizzuwatnien d'Ištar/Šaušga7. KUB 29.4 (CTH 481) ii 43-45 énumère les cités dans lesquelles se trouvait un temple de la déesse de la nuit. I. Wegner a remarqué que chacune de ces villes, à savoir Agadé, Babylone, Suse, Elam et Hursag-kalama, abritait également le culte d'Ištar (Wegner 1981, 164-165). Cette dernière était identifiée à l'astre Vénus. La déesse de la nuit, en tant qu'aspect d'Ištar/Šaušga, pourrait donc elle aussi être représentée par un astre, comme l'a déjà suggéré V. Haas ${ }^{8}$.

${ }^{6}$ Wegner $(1981,164)$. Lebrun $(1976,137$ et 198) avait proposé d'identifier la déesse de la nuit avec Šaušga de Šamuha à cause de la mention DINGIR GE ${ }_{6}$ URu $\breve{S} a m u h a$ dans ce même texte oraculaire KBo 16.97 Vo 13.

${ }^{7}$ Le fait que la déesse de la nuit soit d'origine kizzuwatnienne est clairement indiqué par KUB 32.133 i 1-4 (Kronasser 1963, 58): UMMA ${ }^{\text {DUTU }}{ }^{S L}$ I murši-DINGIR ${ }^{L I M}$ LUGAL GAL DUMU I šuppiluliuma LUGAL GAL UR.SAG / ABBA $=Y A=z a=k a n$ kuwapi Iduthaliyaš LUGAL GAL DINGIR $\mathrm{GE}_{6}$ IŠTU É DINGIR $\mathrm{GE}_{6}$ / URUkizzuwatni arha šarriyet n=anzzan INA URU šamuha / É.DINGIR ${ }^{L M}$ hanti iyat «Ainsi (parle) Mon Soleil Muršili, grand roi, fils (de) Šuppiluliuma, grand roi, héros: lorsque mon parent Tuthaliya, grand roi, a divisé la déesse de la nuit du temple de la ville de Kizzuwatna, il l'a installée dans un nouveau temple dans la ville de Šamuha.»

${ }^{8}$ Haas $(1994,352)$ : selon V. Haas la déesse de la nuit est l'étoile du soir (c'est-à-dire Vénus). G. Beckman a montré que l'effigie de la déesse qui est confectionnée lors du rituel CTH 481 correspond au firmament et à ses lumières (Beckman 2002, 38: la déesse de la nuit est représentée avec «des étoiles d'argent et d'or»). Contra Ünal (1993) qui proposait d'identifier la déesse à la Lamaštu. 
Il me semble que cette identification de la déesse de la nuit avec un astre peut être corroborée par les sources de Mésopotamie ${ }^{9}$. De cette région proviennent en effet plusieurs prières destinées aux «divinités de la nuit» (ilānū mušit $i)$. Elles sont datées de l'époque paléo-babylonienne jusqu'au premier millénaire avant J.-C.10 et indiquent clairement l'identité des divinités de la nuit: ces dernières sont - ou sont représentées par des étoiles, des constellations et des planètes ${ }^{11}$.

Les prières mésopotamiennes aux divinités de la nuit peuvent être récitées dans deux principaux types de contextes. En premier lieu, elles interviennent dans le cadre de rituels exorcistiques destinés à délivrer une personne d'un ensorcellement, de mauvais présages ou de «mauvais rêves» (HUL Ùmeš) ${ }^{12}$. Dans ce cas, on sollicite les divinités de la nuit pour qu'elles soient les intercesseurs du mortel auprès des dieux personnels de ce dernier. En second lieu, ces prières participent à des rituels qui aident une personne à recevoir un message divin, qu'il soit diurne (vision, présage atmosphérique ou d'une quelqu'autre nature) ou nocturne (rêvemessage $)^{13}$. Certains de ces rituels sont de véritables incubations, à savoir des rituels durant lesquels la personne sollicitant un rêve-message dort d'une manière particulière ${ }^{14}$.

9 G. Beckman a mis en lumière les affinités qui existent entre le rituel CTH 481 dédié à la déesse de la nuit et les rituels «babilili» (Beckman 2002, 37). Son interprétation renforce l'hypothèse proposée dans ce présent article, à savoir qu'il pourrait exister une filiation entre la DINGIR $\mathrm{GE}_{6}$ "hittite» et les divinités de la nuit mésopotamiennes.

10 Mayer (1976, 427-429). Butler (1998, 354 et 367).

11 Butler $\left(1998,354\right.$ et 367) ii 52-53: DINGIR meš $\mathrm{GE}_{6}$-ti ina [ ] / [den.bi.luh] ${ }^{{ }} \mathrm{d}_{n i n}$ ?.bi.luh DINGIR ${ }^{\text {meš }} \mathrm{EN}^{\text {meš }} \mathrm{GE}_{6}$-ti mušitu kallatu $k$ [uttumtu] «divinités de la nuit dans [ à savoir Enbiluh], Ninbiluh, divinités, seigneurs de la nuit, Nuit, l'épouse v[oi-

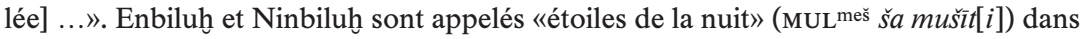
un autre passage du même rituel (ii 44). Cette identification des divinités de la nuit avec des étoiles et planètes est également clairement illustrée dans le texte bilingue hittiteakkadien KUB 4.47 (Houwink ten Cate apud van der Toorn 1985, 125-133). Ce dernier est un rituel mésopotamien contre l'insomnie dans lequel se trouve une prière aux divinités de la nuit (Vo 32-48). Après une longue liste de noms d'astres, l'expression «divinités de la nuit» apparaît deux fois. Certaines de ces divinités sont masculines, d'autres féminines, comme l'indique KUB 4.47 Vo 41: DINGIR MUŠITI ${ }^{\mathrm{D} I S ̌ T A R ~ M U S ̌ I T I . ~}$

12 Prière Maqlû Tablette I 1-5 contre l'ensorcellement (Meier 1937, 7). K. 2315+: 19-109 est une prière aux divinités de la nuit destinée à neutraliser un mauvais présage (Oppenheim 1959).

13 Dans la prière paléo-babylonienne aux divinités de la nuit AO 6769 1. 20-24 (Dossin $1935,181)$, la personne demande aux dieux de lui transmettre un message sollicité (oracle) ou non (présage).

14 C'est en particulier le cas du texte édité par Butler (1998, 353 et 366 ii 44-49). 
Ainsi, tout comme la déesse de la nuit kizzuwatnienne, les divinités de la nuit mésopotamiennes sont associées au phénomène du rêve, ce qui n'est guère surprenant étant donné le caractère nocturne que revêt ce dernier.

\section{Les «mauvais rêves» et les songes du rituel de Walkui}

Quel est le statut des rêves décrits dans le rituel de Walkui (manger une plante urura ou de la viande de porc)? Pour tenter de répondre à cette question, il s'agit de définir la fonction même du rituel. Cette dernière peut être déterminée grâce à l'examen des techniques magiques employées.

La première technique qu'il nous faut considérer est l'utilisation de l'offrande zurkiya. H. Otten (1971, 12-13) a rassemblé les occurrences de ce terme hourrite. Ce dernier étant toujours employé en relation avec le verbe sipant- qui signifie le plus souvent «faire une libation», l'offrande zurkiya est probablement de nature liquide. Elle peut être versée seule ou accompagnée d'un animal sacrifié: une chèvre (KUB 29.4 iii 56), un «oiseau-de-trou» (MUŠEN HUR-RI) ou, comme dans notre texte, un poisson (KUB 29.4 ii 31-32). V. Haas et G. Wilhelm (Haas/Wilhelm 1974, 64f.) ont indiqué que le zurkiya est lié à la notion d'impureté et de faute: zurkiya est directement associé au terme hourrite arni «faute» (de l'akkadien arnu) dans les rituels itkalzi. En outre, il est également souvent accompagné du mot hourrite uziya qui désigne une offrande de viande. C'est la raison pour laquelle E. Laroche $(1973,96-99)$ a proposé de traduire zurkiya par «offrande de sang». Le fait d'utiliser du sang - la substance taboue par excellence (Moyer 1969,69) - au cours d'un rituel a souvent un effet purificateur, comme en témoigne notamment le rituel d'Ulippi dédié à la déesse de la nuit: la statue de la déesse et d'autres éléments liturgiques sont badigeonnés de sang dans le but d'être purifiés ${ }^{15}$.

Par ailleurs, le rituel de Walkui emploie également la fumigation d'encens (Gišlueššar: lignes 10 et 14), geste qui a souvent un rôle cathartique ${ }^{16}$ : les Anciens attribuaient sans doute à la senteur de la fumigation le pouvoir de purifier un lieu, d'une part, et celui d'attirer les dieux d'autre part.

15 Kronasser (1963, 32f. iv 38-39).

16 On comprendra ici «cathartique» dans son sens le plus large, à savoir «purificateur» (voir l'article «cartharsis» de la version informatisée du Trésor de la Langue Française à l'adresse http://frantext.inalf.fr/tlf.htm). 
Par conséquent, le rituel de Walkui semble avoir une fonction cathartique voire exorcistique ${ }^{17}$ : il doit servir à soigner la personne qui s'est vue manger de la viande de porc ou la plante urura en rêve - ou qui a côtoyé l'une ou l'autre de ces choses dans la journée. Mais pourquoi manger de tels aliments était-il considéré comme si dangereux? En quoi un tel songe était-il un «mauvais rêve»?

D'après les textes hittites et mésopotamiens, il semblerait que les Anciens attribuaient trois origines distinctes aux «mauvais rêves»:

1) divine: les rêves-messages défavorables qui prédisent un événement néfaste;

2) démoniaque: les rêves maléfiques d'une part, et les rêves trompeurs d'autre part qui sont envoyés par des fantômes ou des démons;

3 ) humaine et démoniaque simultanément: les rêves impurs qui sont envoyés par des démons mais dans lesquels l'esprit du rêveur participe activement. Cela équivaut à dire que le rêveur est incité par les démons à perpétrer une faute dans son songe ${ }^{18}$.

Je suggère que les rêves "soignés» par le rituel de Walkui soient des rêves impurs, ce qui signifie que la personne commettrait une faute en mangeant de la viande de porc ou une plante urura en rêve. Le nom urura étant un hapax legomenon, on ne peut rien conclure au sujet de cette plante. Mais peut-on penser que la consommation de viande de porc était taboue au Kizzuwatna, région d'où provient ce rituel?

Nous pouvons distinguer trois catégories de tabous alimentaires:

1) une nourriture peut être taboue car elle est considérée comme réservée aux dieux ${ }^{19}$;

2) à l'inverse, elle peut être taboue à cause de son caractère impur;

3) il existe des tabous temporaires, c'est-à-dire des pratiques qui ne

17 C'est également l'interprétation de Lebrun $(1999,607)$.

18 Certains événements rêvés étaient considérés comme aussi tangibles que ceux ayant eu lieu durant la journée. Une faute entraînait les mêmes conséquences qu'elle ait été commise en rêve ou à l'état de veille. La dichotomie rêve-veille $=$ chimère-réalité n'était valable que pour les rêves «quotidiens», c'est-à-dire les songes ne transmettant pas de message divin et ne comportant pas de danger pour le rêveur. Les rêves impurs étaient sans doute considérés comme une sous-catégorie de rêves trompeurs. Les songes de tabous sexuels appartiennent à cet ensemble.

19 Haas $(1994,642)$ cite un passage de la prière de Kantuzzili (KUB 30.10 Ro 12'-13'): $n u$ ANA DINGIR:YA UL kǔšanka linkun lingai=ašta UL kuššanka šarrahhat / šiuni=mi=ma=mu kuit šuppi adanna natta ara n=at UL kǔš̌anka edun «Je n'ai jamais (par)juré vis-à-vis de (toi) mon dieu, je n'ai jamais transgressé de serment, et je n'ai jamais mangé ce qui est sacré pour (toi) mon dieu (et) qu'il m'est interdit de manger.» 
sont interdites que dans certaines occasions et pendant une certaine durée ${ }^{20}$.

Des savants ont argué que le porc était considéré comme un animal impur en Mésopotamie et en Anatolie ${ }^{21}$. Etait-ce vraiment le cas et manger sa chair était-il tabou pour autant?

\section{Le Livre des Rêves néo-assyrien et le porc}

Avant d'explorer les sources anatoliennes, il faut considérer le Livre des Rêves néo-assyrien de Ninive qui fait plusieurs allusions à l'apparition du porc en rêve 22 :

1) Oppenheim (1956, 278 et 323 ligne $\mathrm{x}+16)$ : DIŠ UZU ŠAH SUM-š́́ NU.DÙG.GA UZU «Si on lui [=au rêveur] donne de la viande de porc: ce n'est pas un bon présage.» Cette phrase est précédée par la description de la même situation avec de la chair humaine et de la viande de chien.

2) Le passage Oppenheim $(1956,293 \mathrm{f}$. et 337) envisage le fait que le roi (appelé le «souverain» $\left[\mathrm{BARA}_{2}\right.$ ] tout au long de cette section) mange ou touche de la viande de porc. Malheureusement, seules les protases sont préservées. La mention du porc est toujours précédée ou suivie de celle du chien dans ce paragraphe.

3) Oppenheim (1956, 275 et $319 \mathrm{z}+12 \mathrm{f}$.): [DIŠ KI.MIN]-ma ŠAH imhur-šú / DUMUmeš TUK-ši Š̀̀-šú DÙG- $a b$ «[S'il ditto] et rencontre un porc: il aura des enfants, son coeur sera content.» Ainsi, rencontrer un porc n'est pas chose dangereuse, seul manger sa chair s'avère néfaste. Le même cas est envisagé pour le chien juste avant cette phrase.

4) Oppenheim (1956, 315 iii $x+4 f$.): [DIŠ UZU?] ŠAH KÚ KAM-ti ši-gu-u / šib-sat DINGIR $\mathrm{U}_{4}$.BI GÍD «[S'il (rêve qu')]il mange de la [viande] de porc,

20 van der Toorn $(1985,33)$ a étudié les tabous alimentaires en Mésopotamie qui ne sont appliqués qu'à certaines périodes de l'année.

21 Pour la Mésopotamie, voir van der Toorn $(1985,33)$. Pour l'Anatolie, voir Kümmel (1967, 152): «Zwar ist die Verwendung von Hunden, zumal jungen, im hethitischen Beschwörungsritual nicht ganz so selten, wie O. Masson annahm, doch bilden Hund und Schwein nach Anschauung der Hethiter eine besondere Gruppe als niederste Kategorie der Tierwelt, die normalerweise auch als unrein gelten. Die chthonische Beziehung des Hundes darf dabei wohl auch für das ebenfalls die Erde aufwühlende Schwein gelten.» Ertem (1965, 71-78) a réuni quelques occurrences du sumérogramme ŠAH dans les textes hittites. Voir également la synthèse de Hoffner $(1967,183)$.

22 Ce Livre des Rêves est - du moins en partie - la copie d'un texte plus ancien remontant peut-être à l'époque paléo-babylonienne. En effet, des copies médio-babyloniennes de ce même traité sont connues. Elles possèdent des éléments en commun avec le manuscrit de Ninive (voir Oppenheim 1956, $313 \mathrm{f}$. et Scheil 1913). 
une prière šigu est requise, colère du dieu; se(s) jour(s) seront longs ${ }^{23}$.» La prière šigu est une prière expiatoire ${ }^{24}$. Ce contexte semble être le plus proche de celui du rituel de Walkui.

\section{Le porc dans la religion hittite}

\section{1) La fertilité du porc:}

D'après un texte hittite décrivant un rituel, le porc - et plus particulièrement la truie adulte -, lorsqu'il est enterré sous les fondations d'une demeure, est considéré comme ayant le pouvoir d'apporter la prospérité de celle-ci ${ }^{25}$. D'autres fragments de rituels hittites mentionnent également la fertilité réputée du porc ${ }^{26}$.

2) Le porc et les dieux:

KBo 11.34 i 1-6 (CTH 670) décrit une fête religieuse durant laquelle on tue un porc ${ }^{27}$. Le texte ne précise cependant pas le but de ce sacrifice. $\mathrm{La}$ «fête du porc» intervient lors du trente-cinquième jour de la fête de la hâte; on ne sait malheureusement rien de son contenu ${ }^{28}$.

Plusieurs textes dont un inventaire cultuel et un fragment de description de fête religieuse signalent que des porcs sont offerts à certaines divinités, à savoir: «les dieux des nuages», «le dieu de l'orage des nuages»,

23 L'apparente contradiction qui existe entre la fin de l'apodose «se(s) jour(s) seront longs» et le reste de la phrase s'explique peut-être de la manière suivante: la vie du rêveur sera longue à condition qu'il exécute la prière šigû réclamée par le dieu courroucé.

${ }^{24}$ Concernant les prières šigu, voir Mayer $(1976,111)$ et van der Toorn $(1985,117-121)$.

25 KUB 40.23 et duplicats: Haas (1994, 540 note 9); édition par Kühne (1972, 250f.).

26 Otten/Siegelová $(1970,32)$ : Bo 3617 et duplicats, ligne 4'. Ehelolf $(1933,5)$ et CHD L-N, 112: VAT 13042 Ro 19-22. Les textes mésopotamiens sont encore plus explicites et font allusion à l'exceptionnelle sexualité de cet animal: voir les «chansons d'amour» mésopotamiennes (Cooper 1996, 51 note 16) et les rituels šà.zi.ga contre l'impuissance sexuelle (Biggs 1967, ${ }^{\circ} 12$ ).

27 [ $m$ ] an URu hattušaz / [UR]U dammelha pānzi / INA URU tuggaštuwa 1 ŠAH / hazzianzi takku"ma=aš / UL=ma hapzi ta natta / hazzianzi "[S]i on va de Hुattuša à Dammelha, on tue un porc dans la ville de Tuggaštuwa. Mais s'il [= le porc] ne fuit pas, on ne (le) tue pas.» Le fait que le porc ne fuie pas à l'approche de ces bourreaux semble être vu comme un signe défavorable.

28 Nakamura (2002, 70f.). Ce jour du porc a-t-il une fonction funéraire? Cette fête de la hâte (nuntarriyašhaš) doit être partiellement issue d'une tradition hattie étant donné la présence des prêtresses NIN.DINGIR: ces dernières participent aux fêtes hatto-hittites (Mestieri 420). 
la déesse Soleil de la terre, les dieux Zawalli, le dieu de l'orage de Nerik ou de Tarma/kuliya, Pentaruhšsi, le dieu de l'orage de l'éclair, Hannahanna et les Gulšeš29. Au cours d'un rituel d'Ištanuwa, des porcs sont sa-

29 Inventaire cultuel KUB 12.2 i $16 \mathrm{f}$ :: DINGIR ${ }^{\mathrm{MES}}$ LÚ $^{\mathrm{MES}}$ alpaš ${ }^{\mathrm{NA}} 4 \mathrm{ZI} . \mathrm{KIN}{ }^{\mathrm{I}}$ dudduwalliš [LÚSANGA] / 1 ŠAH.TUR 3 NINDA.GUR 4 .RA 1 DUG KAŠ ANA EZEN $_{4}$ DIŠI «Dieux des nuages: une stèle - Dudduwalli [(est) le prêtre] -, un cochon de lait, trois pains ordinaires (et) un pichet de bière pour la fête du printemps.» Ibid. iii $3 \mathrm{f}$.: ${ }^{\mathrm{D} U}$ alpaš ${ }^{\mathrm{NA}_{4}} \mathrm{ZI}$.KIN 1 ŠAH.TUR NINDA.GUR 4 . RA $=m a / I S ̌ T U$ URU ${ }^{L I M}$ peškanzi 1 DUG KAŠ $A N A$ EZEN $_{4}$ [DIŠI] «Dieu de l'orage des nuages: on donnera une stèle, un cochon de lait et du pain ordinaire de la ville, (plus) un pichet de bière pour la fête du [printemps].» Ibid. iii $13 \mathrm{f}$.: taknaš 'UTU-uš NA4ZI.KIN 1 ŠAH.TUR 3 NINDA.GUR 4 .RA / 1 DUG KAŠ $A N A$ EZEN $_{4}$ DIŠI «Déesse Soleil de la terre: une stèle, un cochon de lait, trois pains ordinaires (et) un pichet de bière pour la

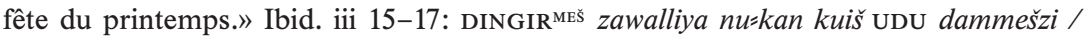
kuišs=ma ŠAH.TUR pē harzi ANA EZEN SIŠI / NINDA.GUR $_{4}$.RA $=m a$ KAŠ IŠTU URU ${ }^{L I M}$ peškanzi «Dieux Zawalli: quelqu'un amènera un mouton et quelqu'un présentera un cochon de lait pour la fête du printemps et on donnera du pain ordinaire et de la bière de la

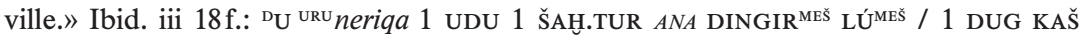

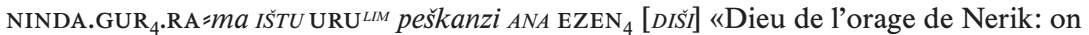
donnera un mouton, un cochon de lait pour les dieux, un pichet de bière et du pain ordinaire de la ville -pour la fête du [printemps].» Ibid. iv $10 \mathrm{f}$.: ${ }^{\mathrm{D} U}{ }^{\mathrm{URU}}$ tarma/kuliya

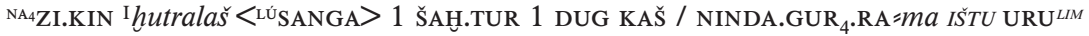
piyanzi ANA EZEN $_{4}$ DIŠI «Dieu de l'orage de Tarma/kuliya: on donnera une stèle Hutrala $<$ (est) le prêtre $>$-, un cochon de lait, un pichet de bière et du pain ordinaire de la ville - pour la fête du printemps.» (pour Tarma/kuliya voir RGTC 6, 407 et 6/2, 162). Ibid. iv 12 f.: ${ }^{\mathrm{D}}$ pentaruhšiš ${ }^{\mathrm{NA}}{ }_{4} Z I$.KIN ${ }^{\mathrm{I}}$ hudrala LÚSANGA 1 ŠAH.TUR / 1 DUG KAŠ NINDA.GUR 4 . RA $=m a$ IŠTU URU ${ }^{L I M}$ peškanzi ANA EZEN $_{4}$ DIŠI «Pentaruhši: on donnera une stèle Hutrala (est) le prêtre -, un cochon de lait, un pichet de bière et du pain ordinaire de la

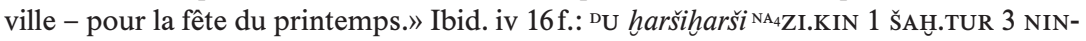
DA.GUR 4 .RA / 1 DUG KAŠ ANA DIŠI «Dieu de l'orage de l'éclair: une stèle, un cochon de lait, trois pains ordinaires et un pichet de bière pour la fête du printemps.» Fragment de texte de fête religieuse KBo 20.89 Ro? 9'f. (Collins 1989, 285 note 917): [...]

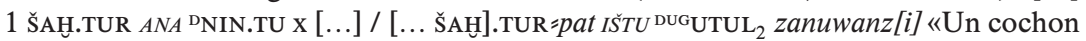
de lait pour Hannahanna [...] on cuit le [porc]elet à l'aide d'un pot.» KBo 11.10 ii 15 'f. (CTH 447: rituel pour les divinités souterraines): 1 ŠAH 1 UDU.NITA 21 GU 4 .APIN.LÁ $\mathrm{SIG}_{5}$-anza NINDA.E.DÉ.A / ki=ma ANA ${ }^{\mathrm{D}} g u l \check{\text { as }}$ š ešdu «Un porc, un mouton mâle, un boeuf de labour en bonne santé, un gâteau à base d'huile, que ces (choses) soient aux Gulšeš!» KUB 55.53 Ro $13 \mathrm{f}$. (fragment de rituel pour Hannahanna): 1 ŠAHु.TUR zeantan $\dot{U}$ 1 DUG KA.GAG GEŠTIN [...] / nu apatta ANA DINGIR.MAH peran tianzi «Un porcelet cuit et un pichet de bière ordinaire (et) du vin [ ] on place cela face à Hannahanna.»

Dans le rituel d'Ašḩella contre une épidémie, le cas est légèrement différent: un bouc et un porc sont sacrifiés pour les dieux des ennemis (Dinçol 1985, 19 et 25). Je ne crois pas que nous devions imaginer qu'il y ait eu un mouton avec le bouc et le porc car deux copies sur trois utilisent le signe NITA $_{2}$ au lieu de UDU. Même si le bouc et le porc sont sacrifiés comme offrande alimentaire pour les dieux, il n'est pas totalement impossible de penser qu'ils étaient utilisés comme substituts: quand un substitut est enterré dans un trou, il sert en même temps d'offrande alimentaire aux divinités sou- 
crifiés à la Porte divinisée ${ }^{30}$. Le rituel vieil-hittite KUB 43.23 destiné à bénir le roi cite parmi les offrandes alimentaires à faire à la terre et à la déesse Soleil de la terre un porcelet (Archi 1979, 34). On remarquera que plusieurs des divinités citées ci-dessus entretiennent une relation avec le monde chtonien. C'est le cas de la déesse Soleil de la terre, des Zawalli, de Huannaḩanna et des Gulšeš.

Un «porc de la divinité Panunta» est même mentionné dans le texte du rituel de Tunnawiya et dans celui du boeuf ${ }^{31}$. Même si l'identité de $\mathrm{Pa}$ nunta n'est pas encore connue, M. Hutter $(1988,123)$ a suggéré que cette divinité soit de nature chtonienne, à cause de l'apparente relation que le texte établit entre le porc et le monde souterrain ${ }^{32}$. Enfin, il existe des rythons en forme de porcs utilisés lors des rituels funéraires royaux (van den Hout 1994, 67) ${ }^{33}$. Le porc est donc le plus souvent mis en relation avec le monde des morts.

\section{3) Le porc comme substitut ${ }^{34}$ :}

Plusieurs rituels exorcistiques anatoliens préconisent le sacrifice puis l'ensevelissement d'un porc dans un trou excavé dans le sol35. C'est le cas du mantalli de Maštigga ${ }^{36}$ et de celui d'Hantitaššu durant lequel l'enseve-

terraines (voir le rituel d'Hुantitaššu mentionné ci-après). Un contexte analogue pourrait être illustré par le rituel contre une malédiction KBo 10.37 iv 32f. (CTH 429): [...] ŠAH.TUR BAL-anti / [...] DINGIR MEš šumeš azzi[k- ...] «On sacrifie [un] porcelet. [ ] vous, les divinités, mang[ez ...]». Le porcelet pourrait constituer l'offrande alimentaire des divinités (souterraines?). Dans le cas du rituel d'Ašḩella, il serait en outre logique de faire appel à des substituts qui absorberaient l'épidémie puis seraient eux-mêmes mangés par les dieux ennemis (ces derniers sont considérés comme étant à l'origine de l'épidémie).

30 KBo 4.11: 9: 2 ŠAH aški apeniššan hattenzi «On tue deux porcs de cette façon pour la (divine) Porte.» (transcr. dans Laroche 1959, 163; HED III, 250 f.). Ištanuwa se situe dans une zone de langue louvite. Forlanini $(1987,115$ note 23$)$ a proposé de la localiser près de Gordion.

31 CTH 760 § 23’: Beckman 1990, 39.

32 Pour les occurrences du «porc de la divinité Panunta», voir Onomasticon I, 348.

33 Le texte d'inventaire KUB 44.6 i 3'f. (Haas 1994, 532 note 322) mentionne deux rythons en bronze en forme de porc ([BI]BRU ŠAH ZABAR $[\ldots]$ / [BI]BRU ŠAH ZABAR [...]). KBo 10.23 v 18'-20' (texte appartenant au corpus de la fête du KI.LAM: Singer (1983-84 II, 14) mentionne également des porcs de cannaie (šAHु.GIš.GI) en or, argent et lapislazuli. Ertem $(1965,73)$ énumère les vases et figurines en forme de porc découverts lors de fouilles archéologiques.

34 Voir également Engelhard (1970, 165-169) et Collins (2002a, 323).

35 Au sujet de ces rituels utilisant un puits funéraire, voir Collins (2002).

${ }^{36}$ CTH 404 ii 45-49 (Rost 1953, 356f.). Pour les éléments culturels kizzuwatniens du rituel de Maštigga, voir Haas/Wilhelm $(1974,46)$. 
lissement du porc est accompagné d'une incantation dédiant ce dernier aux dieux souterrains ${ }^{37}$. Au cours d'un rituel destiné à purifier une armée après une bataille, non seulement un porcelet, mais aussi un chiot et un prisonnier sont coupés en deux ${ }^{38}$. Chacune de leurs moitiés est placée de part et d'autre d'un portail éphémère à travers lequel passe l'armée. Dans le rituel du fleuve de Tunnawi(ya) ${ }^{39}$, le porc est associé au bélier et au chien. Tous trois sont visiblement les substituts du «patient» ${ }^{40}$. Ils sont brûlés par la suite (iii 17 f.). Le fait de brûler un substitut est une des techniques employées pour neutraliser l'impureté que celui-ci a absorbée ${ }^{41}$. Dans le rituel kizzuwatnien d'Allaiturahhi, des figurines de porcs et de chiens sont sans doute utilisées comme substituts ${ }^{42}$.

Ainsi, la majorité des textes de rituels hittites indique que le porc était employé en guise de substitut et offert ou non aux divinités chtoniennes ${ }^{43}$.

${ }^{37}$ KBo 11.14 (CTH 395) iii 5-18: Ünal (1996, 22).

${ }^{38}$ KUB 17.28 iv 45-55 (CTH 426): Masson (1950). D'autres fragments de rituels sont semblables à ce texte: Kümmel (1967, 152-155).

39 CTH 409: Goetze (1938).

40 Il est spécifié que ces animaux doivent être des femelles si le «patient» est une femme et des mâles s'il s'agit d'un homme (i 11-13).

${ }^{41}$ Ce rôle cathartique du feu peut également être relevé dans le rituel de lustration d'une armée déjà mentionné auparavant: chacun de deux côtés du portail éphémère est flanqué d'un foyer. Le rituel d'Ašḩella (CTH 394 - Dinçol [1985] - Vo 1-4) fournit un témoignage analogue. Le porc-substitut peut également être précipité dans le fleuve, comme l'illustre le rituel KUB 36.83+ iv 8' (CTH 433).

42 Haas/Thiel (1978, 106f. ii 29').

43 Hauptmann $(1975,64-67)$. Les fouilles archéologiques de Yazılıkaya ont mis au jour l'offrande d'un porcelet. Cette offrande était sans doute un substitut dédié aux dieux souterrains. Des parallèles palestiniens ont été découverts (de Vaux 1958, 250). Un autre exemple de l'utilisation d'un porc comme substitut se trouve dans le rituel kizzuwatnien «du boeuf» (Beckman 1990).

En Mésopotamie, le porc était utilisé durant les rituels exorcistiques en particulier les šà.zi.g a destinés à soigner l'impuissance sexuelle. Voir par exemple Biggs (1967, $\mathrm{n}^{\circ}$ 27): dans ce rituel šà.zi .g a, on observe le comportement d'un porc face à des figurines. Le fait que l'animal approche de ces dernières indique que la maladie du patient provient d'Ištar (šU dištar «main d'Ištar»), si ce n'est pas le cas, la maladie provient d'un ensorcellement. Le porc servait également de substitut dans les rituels d'exorcisme néo-assyriens contre la Lamaštu. Voir par exemple Myhrman (1902, 154-157). Le porc est également mentionné dans des rituels de magie noire dans lesquels il - ou un chien, un oiseau ou un poisson - mange l'effigie d'une personne pour l'ensorceler (Lambert 1957-58, 292 ligne 25). 


\section{Le porc: un animal impur d'après les textes hittites et mésopotamiens}

1) Un animal impur mais objet de l'élevage:

Aussi bien en Mésopotamie qu'en Anatolie du second millénaire, le porc est élevé, comme l'indiquent non seulement le code d'Hammurapi et les Lois hittites mais aussi la prière de Muršili au dieu Telepinu ${ }^{44}$. Dans cette dernière, chacun des éléments de la vie quotidienne des hommes est mentionné: le grain, la vigne, les arbres fruitiers, les bovins, les ovins, les caprides, les porcs, les mules, les chevaux et l'environnement sauvage $^{45}$. Par conséquent, nous ne pouvons douter du fait que la viande de porc ait été consommée dans ces deux cultures ${ }^{46}$ : en effet, le porc n'a pas d'autre fonction que celle d'animal à viande et à graisse, exceptée celle d'éboueur (il se nourrit des déchets alimentaires humains - Houston 1993, 193 et 199).

Malgré ce fait, les textes mésopotamiens (en particulier ceux du premier millénaire av. J.-C.) font allusion à l'impureté du porc per se. Un proverbe néo-assyrien indique notamment: «Le porc n'est pas pur [...] couvrant de boue son derrière, rendant les rues (mal)odorantes, polluant les maisons. Le porc n'est pas adéquat pour le temple, manque de sens, n'est pas autorisé à errer sur le pavé, une abomination pour tous les dieux, une abomination [pour (son) dieu], maudit par Šamaš.» $)^{47}$ Un recueil de présages mésopotamien indique que quiconque mange de la viande de

44 Il existe des lois hittites concernant le vol d'une truie (Laws $§ 83-86=$ Hoffner 1997, 86-88). Le passage relatif au porc est immédiatement suivi par celui traitant du chien (Laws $\S 87-90=$ Hoffner 1997, 88-90). Une loi concernant le vol d'un porc existe également dans le code d'Hammurapi (Richardson 2000, 44f. L8).

45 CTH 377 ii 14-16: Gurney (1940, 34f.). L'absence du chien est intriguante.

${ }^{46} \mathrm{La}$ quantité de porcs élevés dans une région dépend, entre autres choses, du cadre environnemental: le porc a besoin de beaucoup d'eau pour conserver son corps à température adéquate (Houston 1993, 137). D'autres textes mésopotamiens du second millénaire indiquent que la viande de porc était consommée: TCL 10.45: 4f. (CAD S 104 - texte paléo-babylonien de Larsa), par exemple, «quatre morceaux de viande de porc» sont donnés «en guise de nourriture pour un homme» (4 UzuÚR ŠAH / ana $a[k] a l$ awìlim). Un texte mésopotamien du premier millénaire décrit le traitement qui est prescrit dans le cas où le patient digère mal l'ail, la viande de boeuf, la viande de porc ou la bière (Küchler 1904, 5 f. line $2=$ CAD $\breve{S}_{1}, 104$ ). Pour d'autres attestations, voir Parayre (2000). Concernant des attestations de consommation de lard de porc dans les textes hittites, voir CHD $\breve{S}, 39 \mathrm{c}$.

47 Lambert (1960, 215 lignes 13-16). J'ai préféré traduire lā qašid par «pas pur» au lieu de «unholy» car, outre le fait que qašădum signifierait plutôt «être pur» (AHw. 906), la notion de pureté est plus adéquate dans ce contexte que celle de sacralité: les êtres humains, par exemple, ne sont pas sacrés en eux-mêmes. Cela ne signifie pas pour autant qu'ils soient impurs comme le porc. 
boeuf ou de porc devient impur et n'est plus autorisé à s'approcher du temple 48 .

2) Association entre le porc et le chien dans les textes hittites et mésopotamiens:

Aussi bien dans les textes de rituels hittites que dans les sources mésopotamiennes, l'association entre le porc et le chien est fréquente ${ }^{49}$ : chacun d'eux est manifestement un substitut idéal pour absorber l'impureté d'un mortel ${ }^{50}$. Cette association porc-chien peut également être relevée dans les Lois hittites ${ }^{51}$ et les instructions au personnel des temples hittites. Dans ces dernières, il est stipulé qu'un porc ou un chien était strictement interdit de séjour dans la cuisine d'un temple dans le but de préserver la pureté des pains de sacrifice ${ }^{52}$. Certains comptes-rendus oraculaires hittites signalent qu'un sacrilège (maršaštarri-) a été commis par un porc ou un chien qui a pénétré dans un temple ${ }^{53}$.

3) Pourquoi le porc et le chien sont-ils associés et de caractère impur?:

L'association que les Anciens faisaient entre le porc, le chien et le concept d'impureté peut s'expliquer par plusieurs facteurs:

48 CT 39.38 Vo 11: CAD $\breve{S}_{1}, 104$.

49 Au sujet du chien dans les sources néo-assyriennes, voir Villard (2000).

${ }^{50}$ Les porcs et les chiens qui sont sacrifiés en guise de substituts dans les rituels hittites sont de jeunes animaux. Cela signifie-t-il que les petits de ces deux races étaient considérés comme de meilleurs substituts (Masson 1950, 23) ou bien n'était-ce qu'un simple moyen d'exploiter une progéniture trop nombreuse? Collins $(1990,211)$ pense que ce phénomène peut être expliqué par le fait que les chiots n'avaient aucune valeur «puisqu'ils n'avaient pas encore été dressés en tant que chiens de berger, chiens de garde ou chiens de chasse» (traduit de l'anglais).

51 Hoffner (1997, 157: KBo 6.26 iv 16-19): avoir des rapports sexuels avec un porc et un chien est puni de mort. Les deux animaux sont donc cités côte à côte. Il faut toutefois noter que n'importe quelle pratique zoophile (avec un mouton, une vache, etc.) était punie de la même manière. La spécificité du porc et du chien n'est donc pas prise en compte dans la sentence.

52 KUB 13.4 i 14-20 (Sturtevant/Bechtel 1935, 148f., et Süel 1985, 22f.).

53 KUB 5.9 Ro 28-30 (la scène, décrite par le «chef» des «hommes du palais des grandspères» -c'est-à-dire le temple funéraire - Hellani, se passe dans le temple même, lignes 25f.): ŠAH =wa waštul DÙ-at SISKUR =ma=wa=za UL DÙ-nun / mān kušspat maršaštarraš waš-

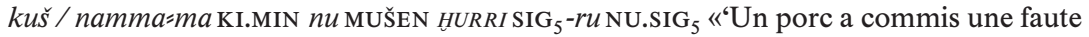
mais je n'ai pas fait de rituel.' Si (ce sont) seulement ces (choses qui) sont des fautes de sacrilège et en outre ditto, que l'oiseau HURRI soit favorable. Défavorable.» Dans KUB 5.10 i $19-23$, un chien a commis un sacrilège équivalent en entrant dans le temple d'Ištar de Ninive (CHD L-N, 198). 
a) Même s'ils sont l'objet d'un élevage - comme c'était le cas en Anatolie, Mésopotamie et même en Syrie, du moins au second millénaire ${ }^{54}$-, ces deux animaux sont souvent considérés comme impurs à cause du fait qu'ils mangent leurs propres excréments et toutes sortes de déchets (qui sont eux-mêmes impurs). KBo 10.37 ii 15-17 indique: «Pour le cheval, l'orge (et) le fourrage mélangé (sont sa nourriture), pour le bétail (c'est) le fourrage (vert), mais pour les chiens (et) les porcs, les déchets (sont) leur nourriture ${ }^{55} . »$

b) Etant donné qu'ils sont toujours en train de gratter le sol, le porc et le chien sont souvent associés au monde souterrain dans les sociétés traditionnelles ${ }^{56}$. Il semble que cela fut également le cas dans le monde anatolien ${ }^{57}$. Selon les Hittites et les Mésopotamiens, le monde souterrain est la principale source de l'impureté, cette dernière se définissant comme l'antithèse de la vie, de la santé et de la pureté58.

c) Une autre source d'impureté est constituée par les relations sexuelles, y compris celles qui ne sont pas considérées comme déviantes. Etant donné qu'aussi bien le porc que le chien sont célèbres pour leur performance sexuelle, leur impureté peut également être attribuée à ce facteur.

d) Le chien et le porc ont un statut spécial dans la société des hommes: ils vivent en communion avec les êtres humains à l'instar des autres

${ }^{54}$ Le fait que le porc ait été élevé, ne serait-ce qu'à une faible échelle, en Syrie ancienne est illustré par un texte de Mari: Durand (1997, 345-347 n²14). Dans ce texte, un chien et un porc sont donnés en pâture à un lion détenu dans un zoo. Une fois de plus, les deux animaux sont associés.

55 CHD L-N, 337: ANA ANŠE.KUR.RA halkiš immiul $A N A \mathrm{GU}_{4}{ }^{\mathrm{MES}}=y a$ / ŠÀ.GAL $A N A$ UR.GI ${ }_{7}^{\mathrm{HA}}$ $\breve{\text { SAAH }}{ }^{\mathrm{HA}}=$ ma mudana / etrešmit. Un autre exemple similaire? provient d'un décret de Hattušili au sujet de la ville de Tiliura (CTH 89: KUB 21.29 iv 6f.): $n u=w a$ UR.[GI 7 TU]R $p a-$ pranza arha:ma=wa / Š 1 MA.[NA] GIšGIGIR ezzai «Le chiot est impur, il mange tout un chariot (d'une valeur) d'une mine.» La traduction et un commentaire de ce proverbe se trouvent dans von Schuler (1965, 148-151). Voir également CHD P, 103. Archi (1988, 27) a mentionné le rôle d'éboueur du porc et du chien.

56 C'est notamment le cas en Grèce où le porc était à l'origine lié au culte de la déesse chtonienne Démeter: il était lancé dans un trou en guise d'offrande à cette divinité (de Vaux 1958, 259). Ce rite fait écho à celui pratiqué lors des rituels de substitutions hittites. Pour une comparaison entre les pratiques grecques et hittites, voir Collins (2002, 235-238).

57 Le caractère chtonien du porc et du chien a déjà été souligné par Kümmel $(1967,152)$, Hoffner (1967a, 400) et Moyer $(1969,96)$.

58 L'impureté se manifeste donc par la maladie et a fortiori par la mort. Cela expliquerait pourquoi les rituels funéraires royaux hittites doivent être pratiqués en cas de "grande faute» (šalliš waštaiš): «faute» serait alors à comprendre comme «impureté» comme Taracha $(2001,690)$ l'a suggéré (contra van den Hout 1995, 195 qui traduit šalliš waštaiš par «grande perte»). 
animaux domestiques (ovidés et bovidés) mais ne sont pas herbivores comme ces derniers (Houston 1993, 199). Pour cette raison, les hommes les ont souvent considérés comme des créatures à part. La meilleure illustration de ce phénomène est la prière de Muwatalli à l'assemblée des dieux dans laquelle le roi demande au dieu Soleil du ciel de délivrer son jugement sur «l'homme, le chien, le porc et les animaux sauvages» $»^{59}$. En outre, en tant qu'animaux omnivores, le chien et le porc peuvent même se nourrir de charognes, ce qui renforce leur relation avec le monde souterrain $^{60}$.

\section{La consommation de viande de porc dans les textes hittites}

1) La viande de porc consommée en milieu religieux hatto-hittite:

Dans la liste de rations KBo 10.31 iii 17'f. de la fête hatto-hittite du KI.LAM, trois porcs sont donnés à un prêtre du dieu de l'orage ${ }^{61}$. Une autre liste de rations vieil-hittite d'une fête religieuse, KBo 20.16++, indique que des porcs sont tués et respectivement donnés à un prêtre hamina et à un «oint» $\left(\text { LÚ GUDU }_{12}\right)^{62}$.

Dans le rituel d'origine hattie KUB 17.28 (CTH 730)63, un cochon de lait est cuisiné puis en partie offert à la déesse Soleil de la terre, en partie mangé par les prêtresses.

59 CTH 381 iii 16f.: Singer (1996, 20 et 39). Güterbock $(1958,242)$ précise que, même si ces prières hittites proviennent de l'héritage mésopotamien, «l'inclusion des animaux, et en particulier du chien et du porc, parmi les personnes que le dieu Soleil juge» est un des «concepts qui est propre aux Hittites».

60 Des malédictions de traités néo-assyriens sont comme suit: «Que les chiens et les porcs mangent votre chair!» (Parpola/Watanabe 1988, 46f. 1. 451: UZU ${ }^{\mathrm{MEš}}-k u n u$ UR.GI ${ }_{7}^{\mathrm{MEs̆}}$ ŠAH $\left.{ }^{\mathrm{ME}} \mathrm{li} k u[l \bar{u}]\right)$.

${ }^{61}$ Singer (1983-84, II, 103): 3 [ŠA] $\mathrm{H}^{\mathrm{HA}} Q A D U$ SU-ŠUNU / LÚ DU dai «L'homme du dieu de l'orage prendra trois [por]cs avec leur chair.»

62 Neu (1980, 38f.) et Singer (1983-84, I 23). La même phrase est répétée plusieurs fois, par exemple dans KBo 20.16 Vo? 9' et 12': [š] AH-ŠUNU kunanzi 1 šAH ANA hami[ni] pianzi «Ils tuent leur(s) porc(s) et on en donne un au hamina.» Une phrase similaire est également présente à plusieurs reprises dans le parallèle KBo 2.12+ (Neu 1980, 44-46), la viande de porc étant cette fois donnée à la vieille femme du palais (ii 7') et à des échansons (Lú.MEšSAGI, ii 8'). Notons que seuls certains membres de l'animal sont donnés dans ce second texte. Je remercie le Dr. B. J. Collins qui m'a indiqué ces deux références. Concernant le hamina, prêtre participant à des cérémonies appartenant à la sphère hattie, voir Mestieri 111-113.

63 Hauptmann $(1975,67)$ et Ertem $(1965,75)$. 
2) Le cas de KBo 3.60:

Le célèbre texte KBo $3.60^{64}$, décrivant des pratiques cannibales, fait également allusion à la viande de porc (ii 12-19):

12. ${ }^{\mathrm{I}}$ kaniuš UZU.ŠAH zeandan dāš

13. tåan IDUMU.MAH.Líl-aš peran daiš

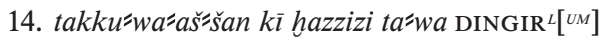

15. takku=wa=aššsan nattasma ha[zzizi]

16. $t a=w a$ antuwahheš $t a=w a \times[$

17. zahhueni IDUMU.MAH.ĹL UZU.šAH [dās]

18. šanap ezta

«Kaniu prit de la viande de porc cuite et la déposa devant DUMU.MAH.LÍL: 'S'il marque cela [=la viande], (il est) un dieu; mais s'il le ne ma[rque] pas, (il est) un être humain, (il est) un [mortel ${ }^{65}$ et] nous [1e] combattrons.' DUMU.MAH.Líl [prit] la viande de porc et la mangea entièrement.»

Le rite décrit dans ce passage est loin d'être clair. Il s'agit d'une sorte de test destiné à déterminer la nature - divine ou humaine - d'un individu. Mais en quoi consistait exactement ce test? Le verbe hazziya - qui est employé dans ce passage a plusieurs sens: il peut signifier «piquer, frapper, percer, atteindre (à l'aide d'une flèche)» ou «marquer, inscrire» ${ }^{66}$. L'objet de ce verbe étant en l'occurrence de la viande, faut-il se résoudre à traduire hazziya- par «marquer»? La marque qu'il s'agirait de faire ou non sur de la nourriture désignerait cette dernière comme impure et donc taboue pour les dieux (mais autorisée pour les hommes). DUMU.MAH.LÍL semble en effet manger la viande, ce qui parait confirmer sa nature humaine. Toutefois, la proposition d'O. Soysal $(1988,119 \mathrm{f}$. et note 41) consistant à reconnaître dans cet emploi de hazziya- un sens abstrait («merken», «treffen», «erkennen») ne peut être entièrement écartée.

Quoi qu'il en soit, ce texte KBo 3.60 pourrait être issu - ne serait-ce qu'en partie - de la tradition littéraire mésopotamienne (Soysal 1988, 109). Il faut donc le considérer avec beaucoup de prudence: comment savoir à quelle culture appartiennent les pratiques qui y sont décrites et ce qu'elles signifient? Quelle est la part du mythe et celle de la réalité dans ce récit qui met en scène les ennemis des Hittites ${ }^{67}$ ?

${ }^{64}$ Güterbock (1938, 104-111). Les références bibliographiques concernant ce texte ont été énumérées dans Soysal (1988). A ces références, il faut à présent ajouter Soysal (1999, 137-145) et de Martino (2002).

65 Proposition de restitution de Güterbock $(1938,107)$.

66 hazziya- a le sens de «marquer, inscrire» dans la Tablette de bronze iv 22 (Otten 1988, 27) et dans KBo 22.1 Ro 23 (CTH 272).

${ }^{67}$ Cette question se pose également pour le cannibalisme qui y est décrit. 
Conclusion sur le rituel de Walkui et la consommation de viande de porc

Le rituel de Walkui semble indiquer que manger - ou simplement rêver que l'on mange - de la viande de porc rendait impur en Kizzuwatna. Certains textes relatifs à des fêtes d'origine hattie semblent indiquer que manger du porc pendant une fête religieuse, et même l'offrir à des divinités non-chtoniennes était envisageable. De ce fait, auquel s'ajoutent les rituels majoritairement kizzuwatniens utilisant le porc comme substitut, faut-il penser que la méfiance envers la viande de porc était plus grande en Kizzuwatna que dans les autres régions du pays hittite?

D'autre part, pourquoi le songe de ce rituel nécessite-t-il d'être exorcisé par un traitement aussi long? Pourquoi une simple purification ne suffit-elle pas? Si le texte avait précisé une circonstance durant laquelle un tel rêve doit être évité, cela aurait expliqué la dangerosité de ce dernier. Mais ce n'est pas le cas. Par conséquent, il nous faut chercher une autre interprétation.

Le texte indique que la personne à soigner a pu voir une plante urura dans un temple. Pour cette raison, peut-être pouvons-nous penser que ce rituel de Walkui était exclusivement destiné à une certaine catégorie de personnes, à savoir les prêtres (de la déesse de la nuit), voire le roi? En effet, les prêtres et le roi entretenaient une relation particulière avec la pureté: ils devaient être purs presque en permanence à cause de leurs contacts fréquents avec les dieux. Ainsi, un rêve impur était bien plus périlleux pour eux que pour le reste de la population.

Par conséquent, même dans les régions où la consommation de viande de porc était considérée comme rendant le mangeur impur - comme cela semble avoir été le cas au Kizzuwatna à l'époque hittite - il n'y avait sans doute pas de tabou alimentaire comparable à celui appliqué dans les religions juive et musulmane actuelles ${ }^{68}$. En effet, exactement comme un homme qui avait un rapport sexuel avec sa femme n'était pas considéré comme ayant commis une faute mais s'était rendu impur, le fait de manger de la viande de porc devait être toléré pour les personnes «normales». La seule règle à respecter alors consistait à se purifier avant de pénétrer dans un lieu sacré - et par conséquent pur, à savoir le temple et certains secteurs du palais.

68 van der Toorn $(1985,34 \mathrm{f}$. notes $338 \mathrm{f}$. avec références bibliographiques): «The 'unclean' animals were not under all circumstances prohibited as food. Although pigs provoked a general disgust, pork was a dish commonly enjoyed.» 


\section{Bibliographie}

Les abréviations employées sont celles de H. G. Güterbock/H. A. Hoffner (éds.), The Hittite Dictionary of the Oriental Institute of the University of Chicago (Chicago 1989-) $(=\mathrm{CHD})$ et d'A. L. Oppenheim et al. (éds.), The Assyrian Dictionary of the Oriental Institute of the University of Chicago (Chicago 1964-) (= CAD).

Archi, A. (1979): Auguri per il Labarna, dans: O. Carruba (éd.), Studia mediterranea Piero Meriggi dicata I. Studia Mediterranea 1 (Parie) 27-51

Archi, A. (1988): Société des hommes et société des animaux, dans: F. Imparati (éd.), Studi di storia e di filologia anatolica dedicati a Giovanni Pugliese Carratelli. Eothen (Florence) 25-37

Beckman, G. (1990): The Hittite «Ritual of the Ox» (CTH 760.I.2-3), Or NS 59, $34-55$

Beckman, G. (2002): Babyloniaca Hethitica: The «babilili-Ritual» from Boğazköy (CTH 718), dans: K. A. Yener/H. A. Hoffner (éds.), Recent Developments in Hittite Archaeology and History. Papers in Memory of Hans G. Güterbock (Winona Lake) 35-41

Biggs, R. D. (1967): ŠÀ.ZI.GA. Ancient Mesopotamian Potency Incantations. TCS 2 (New York)

Butler, S. A. L. (1998): Mesopotamian Conceptions of Dreams and Dream Rituals. AOAT 258 (Münster)

Collins, B. J. (1989): The Representation of Wild Animals in Hittite Texts. PhD Diss. Yale Univ.

Collins, B. J. (1990): The Puppy in Hittite Ritual, JCS 42, 211-226

Collins, B. J. (2002): Necromancy, Fertility and the Dark Earth: the Use of Ritual Pits in Hittite Cult, dans: P. Mirecki/M. Meyer (éds.), Magic and Ritual in the Ancient World. Religions in the Graeco-Roman World 141 (Leyde) 224-241

Collins, B. J. (2002a): A History of the Animal World in the Ancient Near East. HdO 1/64 (Leyde/New York/Cologne)

Cooper, J. S. (1996): Magic and M(is)use: poetic promiscuity in Mesopotamian Ritual, dans: M. E. Vogelzang/H. L. J. Vanstiphout (éds.), Mesopotamian Poetic Language: Sumerian and Akkadian. Proceedings of the Groningen Group for the Study of Mesopotamian Literature 2. CM 6 (Groningen) 47-57

de Martino, S. (2002): The Military Exploits of the Hittite King Hattušili I in Lands Situated Between the Upper Euphrates and the Upper Tigris, dans: P. Taracha (éd.), Silva Anatolica. Anatolian Studies presented to Maciej Popko (Varsovie) 77-85

Dinçol, A. (1985): Ašhella rituali (CTH 394) ve Hititlerde salgın hastalıklara karşı yapılan majik işlemlere toplu bir bakış, Belleten 49/193, 1-40

Dossin, G. (1935): Prières aux «dieux de la nuit» (AO 6769), RA 32, 179-187

Durand, J.-M. (1983): Textes administratifs des salles 134 et 160 du palais de Mari. ARM 21 (Paris)

Durand, J.-M. (1997): Les documents épistolaires de Mari I. LAPO 16 (Paris)

Ehelolf, H. (1933): Heth. milit = «Honig», OLZ 36, 1-7

Engelhard, D. H. (1970): Hittite Magical Practices: an Analysis. PhD Diss. Brandeis Univ.

Ertem, H. (1965): Boğazköy metinlerine göre Hititler devri Anadolu'sunun faunası. Ankara Üniversitesi Dil ve Tarih Fakültesi Yayınları 157 (Ankara)

Forlanini, M. (1987): Un toponyme antique d'origine hattie?, Hethitica 8, 105-122

Goetze, A. (1938): The Ritual of Tunnawi. AOS 14 (New Haven)

Gurney, O. R. (1940): Hittite Prayers of Mursili II. AAA 27 (Liverpool) 
Güterbock, H. G. (1938): Die historische Tradition und ihre literarische Gestaltung bei Babyloniern und Hethitern bis 1200, ZA 44, 45-149

Güterbock, H. G. (1958): The Composition of Hittite Prayers to the Sun, JAOS 78, 237-245

Haas, V. (1994): Geschichte der hethitischen Religion. HdO 1/15 (Leyde)

Haas, V. /Thiel, H. J. (1978): Die Beschwörungsrituale der Allaiturah(h)i und verwandte Texte. AOAT 31 (Neukirchen-Vluyn)

Haas, V. /Wilhelm, G. (1974): Hurritische und luwische Riten aus Kizzuwatna. AOATS 3 (Neukirchen-Vluyn)

Hauptmann, H. (1975): Die Felsspalte D, dans: K. Bittel et al. (éds.), Das hethitische Felsheiligtum Yazllıaya. BoHa 9 (Berlin)

Hoffner, H. A. (1967): Compte rendu d'E. von Schuler, Die Kaskäer (1965), JAOS 87, $179-185$

Hoffner, H. A. (1967a): Second Millennium Antecedents to the Hebrew 'ÔB, JBL 86, 385-401

Hoffner, H. A. (1997): The Laws of the Hittites. A critical Edition. DMOA 23 (Leyde/ New York/Cologne)

Hoffner, H. A. (1998): Hittite Myths. $2^{\text {nd }}$ ed. SBL Writings from the Ancient World Series (Atlanta)

Houston, W. (1993): Purity and Monotheism. Clean and Unclean Animals in Biblical Law. Journal for the Study of the Old Testament Supplement Series 140 (Sheffield)

van den Hout, T. (1994): Death as a Privilege. The Hittite Royal Funerary Ritual, dans: J. M. Bremer/T. van den Hout/R. Peters (éds.), Hidden Futures. Death and Immortality in Ancient Egypt, Anatolia, the Classical, Biblical and Arabic-Islamic World (Amsterdam) $37-75$

van den Hout, T. (1995): An Image of the Dead? Some Remarks on the Second Day of the Hittite Royal Funerary Ritual, dans: O. Carruba et al. (éds.), Atti del II congresso internazionale di Hittitologia. Studia Mediterranea 9 (Pavie) 195-211

Hutter, M. (1988): Behexung, Entsühnung und Heilung. Das Ritual der Tunnawiya für ein Königspaar aus mittelhethitischer Zeit (KBo XXI 1-KUB IX 34-KBo XXI 6). OBO 82 (Göttingen)

Kronasser, H. (1963): Die Umsiedelung der schwarzen Gottheit. Das hethitische Ritual KUB XXIX 4 (des Ulippi). Österr. Akad. der Wiss., Philos.-hist. K1. Sitzungsber. 241/3 (Vienne)

Küchler, F. (1904): Beiträge zur Kenntnis der assyrisch-babylonischen Medizin. Assyriologische Bibliothek 18 (Leipzig)

Kühne, C. (1972): Bemerkungen zu kürzlich edierten hethitischen Texten, ZA 62, 236-261

Kümmel, H. M. (1967): Ersatzrituale für den hethitischen König. StBoT 3 (Wiesbaden)

Lambert, W. G. (1957-58): An Incantation of the Maqlû Type, AfO 18, 288-299

Lambert, W. G. (1960): Babylonian Wisdom Literature (Oxford)

Laroche, E. (1959): Dictionnaire de la langue louvite. Bibliothèque archéologique et historique de l'institut français d'archéologie d'Istanbul 6 (Paris)

Laroche, E. (1973): Etudes de linguistique anatolienne, RHA 31, 83-99

Lebrun, R. (1976): Samuha, foyer religieux de l'empire hittite. Publication de l'Institut Orientaliste de Louvain 11 (Louvain-La-Neuve)

Lebrun, R. (1980): Hymnes et Prières Hittites. Homo Religiosus 4 (Louvain-La-Neuve)

Lebrun, R. (1999): Fragment d'un rituel de Walkui, prêtre de la déesse de la nuit (KBo XXXII $176=$ Bo 83/902), ArOr 67/4, 601-608

Masson, O. (1950): A propos d'un rituel hittite pour la lustration d'une armée: le rite de purification par le passage entre les deux parties d'une victime, RHR 137, 5-25 
Mayer, W. (1976): Untersuchungen zur Formensprache der babylonischen «Gebetsbeschwörungen». Studia Pohl s. m. 5 (Rome)

Meier, G. (1937): Die assyrische Beschwörungssammlung Maqlû, AfO Beiheft 2 (Vienne)

Moyer, J. C. (1969): The Concept of Ritual Purity among the Hittites. PhD Diss. Brandeis Univ.

Myhrman, D. W. (1902): Die Labartu-Texte. Babylonische Beschwörungsformeln nebst Zauberverfahren gegen die Dämonin Labartu, ZA 16, 141-200

Nakamura, M. (2002): Das hethitische nuntarriyašha-Fest. PIHANS 94 (Leyde)

Neu, E. (1980): Althethitische Ritualtexte in Umschrift. StBoT 25 (Wiesbaden)

Onomasticon: B. H. L. van Gessel, Onomasticon of the Hittite Pantheon. HdO 1 / 33. (Leyde 1998-2001)

Oppenheim, A. L. (1956): The Interpretation of Dreams in the Ancient Near East. With a translation of an Assyrian Dream-Book. Transactions of the American Philosophical Society NS 46/3 (Philadelphie)

Oppenheim, A. L. (1959): A New Prayer to the «Gods of the Night», Studia Biblica et Orientalia III. AnBi 12, 282-301

Otten, H. (1971): Materialien zum hethitischen Lexikon. StBoT 15 (Wiesbaden)

Otten, H. (1988): Die Bronzetafel aus Boğazköy. Ein Staatsvertrag Tuthaliyas IV. StBoT Beiheft 1 (Wiesbaden)

Otten, H. /Siegelová, J. (1970): Die hethitischen Gulš-Gottheiten und die Erschaffung der Menschen, AfO 23, 32-38

Parayre, D. (2000): Les suidés dans le monde syro-mésopotamien aux époques historiques, dans: Les animaux et les hommes dans le monde syro-mésopotamien aux époques historiques. Topoi suppl. 2, 141-206

Parpola, S. /Watanabe, K. (1988): Neo-assyrian Treaties and Loyalty Oaths. SAA 2 (Helsinki)

Richardson, M. E. J. (2000): Hammurabi's Laws. Text, Translation and Glossary. The Biblical Seminar 73. Semitic Texts and Studies 2 (Sheffield)

Rost, L. (1953): Ein hethitisches Ritual gegen Familienzwist, MIO 1, 345-379

Salvini, M. /Wegner, I. (1986): Die Rituale des AZU-Priesters. ChS 2 (Rome)

Scheil, V. (1913): Textes élamites-sémitiques. MDP 14 (Paris)

von Schuler, E. (1965): Die Kaškäer. Ein Beitrag zur Ethnographie des alten Kleinasien (Berlin)

Singer, I. (1983-84): The Hittite KI.LAM Festival. StBoT 27-28 (Wiesbaden)

Singer, I. (1996): Muwatalli's Prayer to the Assembly of Gods through the Storm-God of Lightning (CTH 381) (Atlanta)

Soysal, O. (1988): Einige Überlegungen zu KBo III 60, VO 7, 107-128

Soysal, O. (1999): Beiträge zur althethitischen Geschichte (I). Ergänzende Bemerkungen zur Puhanu-Chronik und zum Menschenfresser-Text, Hethitica 14, 109-145

Sturtevant, E. H. / Bechtel, G. (1935): A Hittite Chrestomacy. William Dwight Whitney Linguistic Series (Philadelphie)

Süel, A. (1985): Hitit kaynaklarında tapınak görevlileri ile ilgili bir direktif metni. Ankara Üniversitesi Dil ve Tarih-Coğrafya Fakültesi Yayınları 350 (Ankara)

Taracha, P. (2001): Aspekte der Hurritisierung Kleinasiens: Ein Beschwörungsritual aus mittelhethitischer Zeit, dans: G. Wilhelm (éd.), Akten des IV. Internationalen Kongresses für Hethitologie (Würzburg, 4.-8. Oktober 1999). StBoT 45 (Wiesbaden) 685-695

van der Toorn, K. (1985): Sin and Sanction in Israel and Mesopotamia. A comparative study. Studia Semitica Neerlandica (Assen) 
Ünal, A. (1993): The Nature and Iconographical Traits of «Goddess of Darkness», dans: M. J. Mellink et al. (éds.), Aspects of Art and Iconography: Anatolia and its Neighbors. Studies in Honor of Nimet Özgüç (Ankara) 639-644

Ünal, A. (1996): The Hittite Ritual of Hantitaššu from the City of Hurma against troublesome years. Publications of Turkish Historical Society Serial VI n ${ }^{\circ} 45$ (Ankara)

de Vaux, R. (1958): Les sacrifices de porcs en Palestine et dans l'Orient ancien, dans: J. Hempel/L. Rost (éds.), Von Ugarit nach Qumran. Beiträge zur alttestamentlichen und altorientalischen Forschung Otto Eissfeldt (Berlin) 250-265

Villard, P. (2000): Le chien dans la documentation néo-assyrienne, dans: Les animaux et les hommes dans le monde syro-mésopotamien aux époques historiques. Topoi suppl. 2, 235-249

Wegner, I. (1981): Gestalt und Kult der Ištar-Šawuška in Kleinasien. Hurritologische Studien III. AOAT 36 (Neukirchen-Vluyn) 